The effect of large and small magnitude of intertrial reinforcement on successive

\section{contrast effects*}

\author{
RICHARD S. CALEF† \\ Southern Illinois University, Carbondale, Ill. 62901
}

To investigate the effect of magnitude of intertrial reinforcement (ITR) on successive contrast effects, a total of 128 albino rats was assigned to 32 groups to form a Preshift ITR by Postshift ITR by Preshift Incentive by Postshift Incentive by ITR-Incentive Interval factorial design. Of major importance was the observation that "elation" and "depression" effects occurred solely for Ss receiving a large preshift magnitude of ITR. The results were explained in terms of frustration theory.

The research in instrumental conditioning is replete with data showing the effect of shift in reward magnitude (Black, 1968). Specifically, many studies (e.g., Crespi, 1942; Gonzales et al, 1962; Capaldi \& Lynch, 1967; Ehrenfreund, 1971) have shown that Ss shifted from large to small magnitude of reward run more slowly than Ss maintained on small magnitude of reward, a phenomenon designated as the "depression" effect. Theories based on Amsel's (1958) frustration theory are perhaps the most easily applied incentive-shift formulations. Such an approach can readily account for the developing "depression" effect as a consequence of conditioned interfering responses elicited by frustration stimuli, which, in turn, result from a shift from large to small magnitude of reward.

The results reported by Davis \& North (1967) were particularly supportive of a frustration interpretation of the "depression" effect. In the study, Ss were trained on a varied large and small magnitude of reward schedule in the runway and were shifted to consistent small magnitude of reward. These Ss did not run more slowly than a group of Ss maintained on small magnitude of reward. A frustration interpretation would predict the latter finding because shifted Ss should have learned to run in the presence of frustration

*This study is based on a dissertation submitted to the graduate school, Southern Illinois University, in partial fulfillment of the requirements for the $P h D$ degree. The research was supported by Research Grant MH10340 from the United States Public Health Service. The author gratefully acknowledges the guidance and encouragement provided by James $H$. McHose, chairman of his dissertation committee.

†Now at West Virginia Wesleyan College. Requests for reprints should be sent to Richard S. Calef. Department of Psychology, West Virginia Wesleyan College, Buckhannon. W. Va. 26201. cues (rf-sf) that result from repeated experience with large and small magnitudes of reward during preshift training. Following the shift to consistent small magnitude of reward, Ss should have continued to respond well in the presence of frustration stimuli.

In a study by Capaldi et al (1970), the result that Ss given only goalbox placement during acquisition showed a PREE in the runway implies that Ss make an instrumental response during frustration stimuli evoke these instrumental responses. The present study tested the hypothesis that an absence of the "depression" effect should also occur in a situation in which Ss receive small magnitude of reward following placement in the goalbox (ITR) and large magnitude of reward following a runway response during preshift training. If Ss are emitting a fractional running response in the goalbox, then frustration theory predicts that $S s$ receiving large magnitude of runway reward/small magnitude of ITR during preshift and small magnitude of reward in the runway during postshift should show no "depression" effect, whereas Ss receiving large magnitude of runway reward/large magnitude of ITR during preshift and small magnitude of reward in the runway during postshift should show a "depression" effect.

The present study also tested the effects of large and small magnitude of ITR on the elusive "elation" effect produced by an upward shift in magnitude of runway reward. Incidentally, the study also investigated the effects of the temporal interval between ITR and response measurement.

\section{SUBJECTS}

The Ss were 128 experimentally naive male albino rats, approximately 110 days old at the beginning of the experiment. The rats were obtained from the Holtzman Co., Madison, Wisconsin. goalbox placement and that
APPARATUS

The runway apparatus was essentially the same as that used by Ludvigson \& Gay (1966), except that only one of the multiple parallel alleys was used. The alley was comprised of a $33.02 \mathrm{~cm}$ white startbox, a $66.04-\mathrm{cm}$ white run section, and a $30.48 \cdot \mathrm{cm}$ white goalbox. The inner width and height of each section of the runway was $7.62 \mathrm{~cm}$. Photocell and clock circuitry provided independent traversal times over the first $20.32 \cdot \mathrm{cm}$ segment (start time), the second $30.48-\mathrm{cm}$ segment (run time), and the third 20.32-cm segment (goal time) of the alley. The apparatus also contained a solenoid-operated opaque guillotine-type retrace door separating goal from alley section and an opaque Plexiglas ceiling. Air was exhausted from the alley by means of a Dayton $(1.5-\mathrm{V})$ blower mounted on the rear of the goalbox. Fresh air was drawn into the apparatus around a Dale chemical deodorant cake mounted on the startbox.

\section{PRELIMINARY TRAINING}

Fourteen days prior to the first day of training (Day 15), all Ss were placed on a 23-h food-deprivation schedule, which was maintained throughout the study. On Days 7-14, Ss were taken from the individual home cages and handled for about $2 \mathrm{~min}$ each. On Days 13 and 14 , Ss were placed in individual carrying cages and taken to the experimental room, where they were allowed to explore start and run sections of the apparatus while clocks, photocells, and doors were operated. On these days, approximately $1 \mathrm{~g}$ of 45-mg Noyes pellets, identical to the subsequent reinforcement pellets, were incorporated into Ss' daily food rations.

\section{EXPERIMENTAL DESIGN} AND PROCEDURE

All Ss received 36 preshift and 16 postshift trials at the rate of 1 trial per day. During the preshift period, the design consisted of a 2 by 2 by 2 factorial, with 16 Ss randomly assigned to each level of each factor. The factors were incentive, 16 or 2 $45-\mathrm{mg}$ pellets received in the goalbox following each traversal of the alley; intertrial reinforcement (ITR), 16 or 2 pellets received upon placement in the goalbox; and the temporal interval between goalbox placement and trial administration (groups), $24 \mathrm{~h}$ or $4 \mathrm{~min}$. Following preshift training, four Ss from each of the eight preshift conditions were assigned randomly to 32 groups to form a 2 (preshift incentive) by 2 (preshift ITR) by 2 (postshift incentive) by 2 (postshift ITR) by 2 (groups) factorial design.

Trials were administered to eight squads of 16 Ss earh, with each $S$ representing a different group. The running order of Ss within a squad was 
LARGE PRESHIFT IT R SMALL PRESHIFT I T R

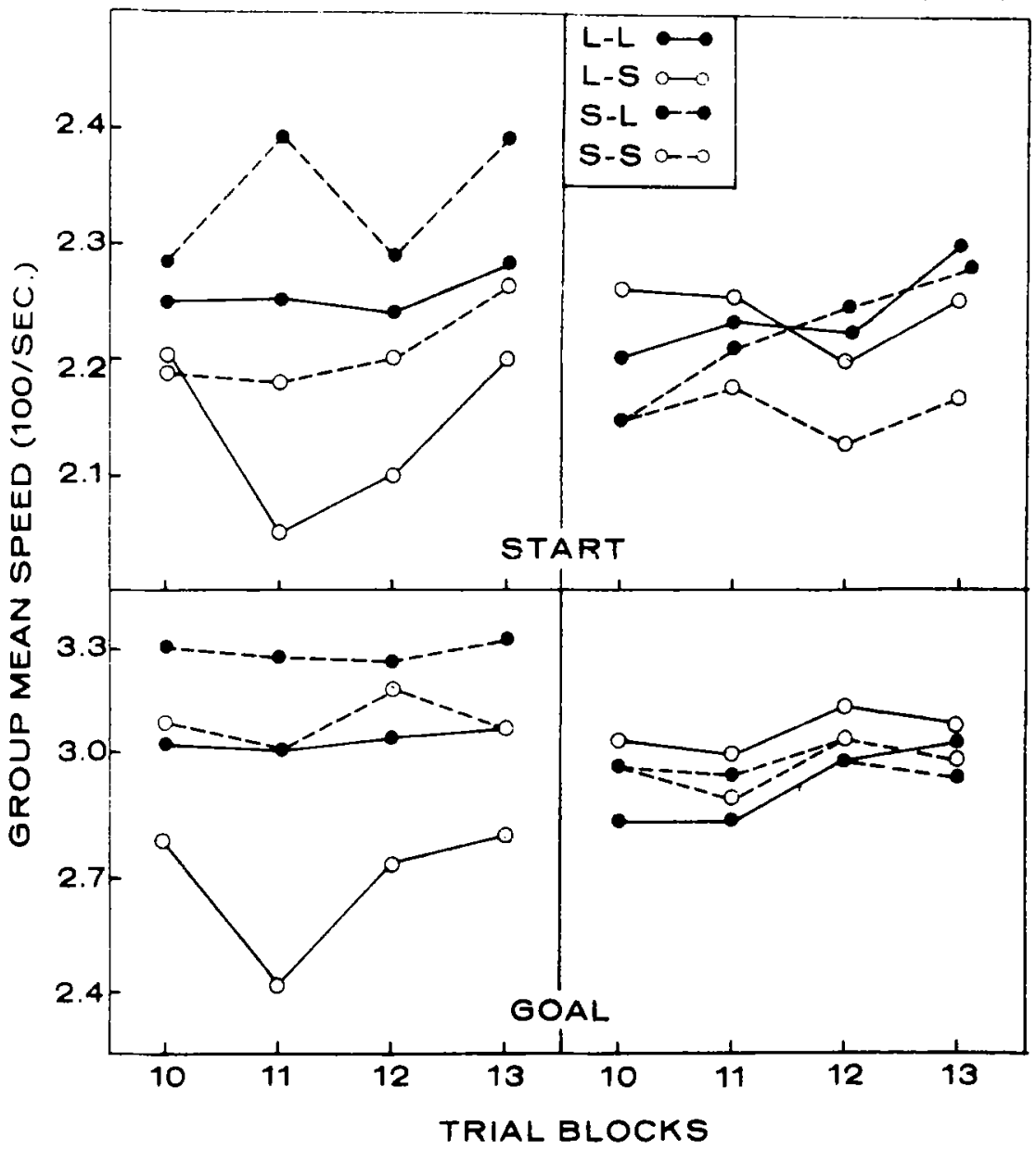

Fig. 1. Group mean postshift start and goal speeds of the incentive data for Ss receiving large preshift magnitude of ITR (left frame) and Ss receiving small preshift magnitude of ITR (right frame).

randomized from day to day. In each squad, 8 of the Ss initially received their ITR (4-min group) but did not receive their running trials until 8 of the other $S s$ in the squad completed their running trials (approximately 3-4 min I. The 8 Ss that initially received their running trials $(24-\mathrm{h}$ group) were placed in their carrying cages following completion of their running trial but did not receive their ITRs until the other 8 Ss completed their running trials (approximately 3-4 min). A running trial was initiated by placing an $S$ in the startbox. After a 3 -sec orientation, the start door was opened and, following S's entry to the goalbox, the retrace door was dropped.

Start, run, and goal times were converted to reciprocals, yielding start, run, and goal speeds, respectively.

\section{RESLLTS}

During both the preshift and postshift periods, the run measure yielded results similar to the start data; therefore. orilv the start and anal data

we presented. Neither the grou variable nor the postshift ITR variable nor interactions of these variables with other variables had statistically significant effects on either preshift or postshift performance. The data to be presented are thus collapsed across the groups and postshift ITR factors.

During the terminal preshift period, Ss receiving large incentive ran faster than Ss receiving small incentive. Three-factor (Incentive by ITR by Groups) analyses of variance performed on the data from the last eight trials yielded a significant incentive effect in the start segment only, $F(1,120)=8.44, p<.01$, and a significant Incentive by ITR interaction in the goal segment only, such that the incentive effect occurred only for $S$ seceiving a small magnitude of ITR, $\mathrm{F}(1,120)=6.85, \mathrm{p}<.01$. Also, Ss receiving large magnitude of ITR ran faster than Ss receiving small magnitude of ITR during the terminal preshift period. Three-factor analyses of variance of the data from the last eight trials yielded a significant ITTR eflect in the goal measure only, $F(1,120)=4.13, p<.05$.

The results during the postshift period are represented in Fig. 1, in which the incentive data are subdivided according to whether Ss received a large or small magnitude of ITR in the preshift period. As can be seen in Fig. 1, Ss shifted from large to small incentive (L-S) ran more slowly than Ss maintained on small incentive (S-S) only when a large magnitude of ITR was presented during the preshift period. Also, Ss shifted from small to large incentive (S-L) ran faster than Ss maintained on large incentive (L-L) only when a large magnitude of ITR was experienced during the preshift training. Five-factor (Preshift Incentive by Postshift Incentive by Preshift ITR by Postshift ITR by Groups) analyses of variance of the data over Blocks 10 and 11 combined yielded a marginally significant start and a significant goal Preshift Incentive by Preshift ITR interaction, $F s(1,96)=4.98$ and 7.05 , respectively, $p<.01$, and a significant Postshift Incentive by Preshift ITR effect for both measures, $\operatorname{Fs}(1,96)=$ 7.76 and 7.45 , respectively, $p<.01$ In order to interpret the interactions in terms of contrast effects, paired comparison tests were performed. Paired comparison tests of the large magnitude of ITR data at Blocks 10 and 11 showed that the S.L groups ran significantly faster than the L-L groups in the start and goal measures, $t(30)=$ 2.15 and $2.73, p<.025$ and $p<.01$ whereas the L-S groups ran significantly more slowly than the S-S groups in both measures, $\mathrm{t}(32)=2.12$ and $6.44, p<.025$ and $p<.01$. Paired comparison tests of the small magnitude of ITR data yielded no significant contrast effects. DISCUSSION
One aspect of the preshift data is worth noting. The observation that Ss that received small magnitude of reward following a runway response during the preshift period ran more slowly than Ss that received large magnitude of reward is consistent with other data (e.g., Kling, 1956; Metzer, Cotton, \& Lewis, 1957; Beier, 1958).

During postshift training, a "depression" effect was present only when Ss received a large magnitude of ITR during preshift training. This finding supports the results of Davis \& North (1967) and frustration theory if it is assumed that $S s$ are being contingently reinforced for a fractional running response following a goalbox placement. Ss receiving small magnitude of ITR and large magnitude of runway reward during preshift training apparently learned 10 run in the presence of frustration cues. Following a shift to small magnitude 
of runway reward, the Ss continued to run well in the presence of frustration stimuli and did not run more slowly than the control group maintained on small magnitude of reward in the runway. However, Ss that received large magnitude of ITR and large magnitude of runway reward during preshift training showed a "depression" effect following a shift to small magnitude of runway reward, possibly because these $S$ s initially experienced primary frustration during postshift training, which, in turn, caused conditioned interfering responses elicited by frustration stimuli.

The present study also investigated the effects of large and small magnitudes of ITR on an upward shift i: magnitude of runway mad. The present resuits showed that ss shifted from small to large magnitude of runway reward ran faster than Ss ramintained on large magnitude of runway reward ("elation": effect) only when large magnitude oi ITR, was experienced during the preshitt period. This observation is of particular interest, since previous literature has shown that the "elation" effect is, at best, elusive (Dunham, 1968; Black, 1968). The present data suggest that "elation" effects are no less genuine than "depression" effects. A possible explanation of the presence and absence of the current "elation" effect may also be derived from frustration theory. Ss that received large magnitude of ITR and small magnitude of runway reward during preshift training possibly learned to run in the presence of frustration stimuli. Following a shift to large magnitude of runway reward, the Ss were motivated, at least temporarily, by $\mathrm{rg}$-sg and $\mathrm{rf}-\mathrm{sf}$, and this produced greater responding than was produced by a control group, which, at the time, was motivated by $\mathrm{rg} \cdot \mathrm{sg}$ only. In other words, the additional motivation provided by $\mathrm{rf}$-sf might have been just what the experimental group needed to enhance performance compared to a control group. Ss that received small magnitude of ITR and small magnitude of runway reward during preshift training did not show an "elation" effect following a shift to large magnitude of runway reward, possibly because these Ss were motivated only by rg-sg, since no frustration occurred for this group. Further research should be done to test the latter explanation of the presence and absence of the current "elation" effects. For example, s trained on variable small and large magnitude of runway reward during preshift training and later shifted to large magnitude of reward should run faster than a control group maintained on consistent large magnitude of reward.

In addition to a frustration interpretation, the results of the present investigation can be discussed in terms of Capaldi's (1967) stimulus a ftereffects theory. According to Capaldi (1967), the reward magnitude received on any trial may provide magnitude-specific stimuli present when the organism responds on the next trial. In this context, the observation that Ss shifted from large (L) to small (S) reward ran more slowly than Ss that always received small reward may be interpreted as a stimulus generalization decrement phenomenon. Thus, Ss shifted from large to small reward should run more slowly than control Ss, because the novel aftereffeet of the new reward for experimental Ss has not had as miluch habit strength accrued to it as has the aftereffect present for small reward control Ss. Alson. within the same context, Ss shifted from small to large reward snould run more slowiy than control Ss maintained on large reward. In regard to the present investigation, Capaldi's aftereffect notion does not prove to be fruitful in the interpretation of the phenomena reported in the study. First, the fact that an "elation" effect occurred following an upward shift in reward is not in accordance with a stimulus aftereffect notion. Secondly, the fact that following a downward shift in reward a "depression" effect occurred only for Ss receiving a large magnitude of preshift ITR is also not in accordance with Capaldi's theory. If an ITR was providing the stimulus for the subsequent running trial, as it has been shown to do in previous studies (e.g., Capaldi, Hart, \& Stanley, 1963; Capaldi \& Spivey, 1964), then Capaldi's aftereffect notion would predict a Preshift ITR (stimulus) by Postshift ITR (stimulus) interaction, such that a change in the stimulus value should produce poorer performance than no change in the stimulus value (i.e., $L-S$ and $S-L<S-S$ and L-L). Specifically, a "depression" effect should occur only when there is a downward shift in the stimulus value from preshift to postshift training, regardless of the magnitude of reward present in the runway. Therefore, the present finding that a "depression" effect occurred solely for Ss receiving a large preshift magnitude of ITR and a shift from large to small runway reward from preshift to postshift training irrespective of their postshift ITR values is nonsupportive of an aftereffect notion. However, there is evidence which indicates that with repeated presentation ITR ceased to effectively replace the aftereffect of the preceding trial, because Ss discriminated between ITRs inirl regular reinforcement ispence. Platl. \& Uatsumoto, 1965). Since the present study involved a relutively large number of ITRs, it seems quite possible that at some point such ITRs ceased to be effective in replacing the aftereffect of the preceding trial. If so, runway reinforcement rather than ITR may have been providing the stimulus value for $S s$ in the present study. Under such conditions, aftereffect theory would predict successive contrast effects following an appropriate shift in runway reinforcement, regardless of the particular magnitude of ITR. In essence, a strict stimulus aftereffect notion fails to explain the present firdings, whether or not it is assumed that $S$ s discriminated between ITRS and regular reinforcement.

\section{AMSEL. A. The role of pustrative} nonreward in noncontinuous reward situations. Psycholoxical Bulletin, 1958. $55,102-119$

BEIER. E. M. Effects of trial-to-tria variation in magnitude of reward upon an instrumental running response. PhD dissertation. Yale University, 1958.

BLACK, $R$. $W$. Shifts in magnitude of reward and contrast effects in instrumental and selective learning: $A$ reinterpretation. Psychological Review. $1968,75,114-126$.

CAPALDI, E. J. A sequential hypothesis of instrumental learning. In $\mathbf{K}$. W. Spence and J. T. Spence (Eds.), The psychology of learning and motivation. Vol. 1. New York: Academic Press. 1967.

CAPALDI, E. J.. CAPALDI, E. D.. \& KASSOVER, K. An instrumental partial reinforcement effect in the absence of any overt instrumental acquisition training. Psychonomic Science, 1970. 21. 145-147.

CAPALDI, E. J.. HART, D., \& STANLEY. L. R. Effect of intertrial reinforcement on the after-effect of nonreinforcement and resistance to extinction. Journal of Experimental Psychology. 1963. 65. 70-74.

CAPALDI, E. J., \& LYNCH, A. D. Iagnitude of partial reward and resistance to extinction: Effect of $X \cdot R$ transitions. Journal of Comparative \& Physiological Psychology, 1968, 65. $179-181$.

CAPALDI, E. J., \& SPIVEY, J. E. Intertrial reinforcement and aftereffects at 24-hour intervals. Psychonomic Science. 1964. 1. $181-182$.

CRESPI, L. P. Quantitative variation in incentive and performance in the white rat. American Journal of Psvehology. $1942,55,467-517$.

DAVIS, S. F., \& NORTH, A. J. The effect of varied reinforcement training on behavior following incentive reduction. Psychonomic Science, 1967, 9, 395-396.

DUNHAM, P. J. Contrasted conditions of reinforcement: A selective criticue. Psychological Bulletin, 1968. fit. 295-315.

EHRENFREUND. D. Effect of drive,$n$ successive magnitude shift in rats. Joum 1 l of Comparative \& Physiolofirit Psychology, 1971, 76, 418-423.

GONZAIES. R. C.. GLEITMAN, H.. \& BITTERMAN, M. E. Some observations on the depression effect. Journal $n$ : Comparative \& Phvsiological Psicholo: $1962.55,578-581$ 

KLING, J. M. Speed of running as a
function of goalbox behavior. Journal of Comparative \& Physiological Psychology. $1956,49,474-476$.

LUDVIGSON, H. W., \& GAY, S. Differential reward conditioning: $S-$ contrast as a function of the magnitude of $\mathrm{S}+$. Psychonomic Science, 1966,5 , 289-290.

METZER, R., COTTON, JW W LEWIS

D. J. Effect of reinforcement magnitude and order of presentation of different magnitudes on runway behavior. Journal of Comparative \& Physiological
Psychology, 1957,50, 184-188 SPENCE, K. W., PLATT, J. R.,

MATSUMOTO, R. Intertial reinforcement and the partial reinforcement effect as a function of number of training trials. Psychonomic Science, 1965, 3, 205-206. 